\section{Risk factors for tuberculosis in contact investigations in Rotterdam, the Netherlands}

\author{
Jesse Eduard Verdier, ${ }^{1,2}$ \\ Sake Jan de Vlas, ${ }^{1}$ \\ Inge D. Kidgell-Koppelaar, ${ }^{2}$ \\ Jan Hendrik Richardus ${ }^{1,2}$ \\ 'Department of Public Health, Erasmus \\ MC, University Medical Center \\ Rotterdam; ' ${ }^{\text {Department }}$ of Infectious \\ Disease Control, Municipal Public Health \\ Service Rotterdam-Rijnmond, Rotterdam, \\ The Netherlands
}

\section{Abstract}

Contact investigations around tuberculosis patients enable early detection of infection and disease, and prevention of secondary tuberculosis cases. We aim to identify risk factors for M. tuberculosis transmission to contacts of tuberculosis patients, based on unique data from routine contact investigations by the Public Health Service in Rotterdam, the Netherlands, collected between 2001 and 2006. Through logistic regression analysis, we determined the effect of various risk factors on the chance of finding a latent tuberculosis (TB) infection or overt tuberculosis case among contacts. A total of 1165 index patients with active tuberculosis were registered and at least one contact was investigated in 731, resulting in 21,540 contacts overall. Altogether, the contact investigations led to 91 cases of active tuberculosis. Of the 12,698 contacts eligible for screening by tuberculin skin test, 1091 (9\%) were diagnosed with latent tuberculosis infections. Risk factors were old age of the contact, old age of the index patient, and the relationship to the index. A larger fraction of infected close contacts was strongly associated with infections among more distant contacts.

Our findings emphasize the importance of including these personal and interpersonal risk factors in decision making in contact investigations.

\section{Introduction}

Contact investigation around tuberculosis (TB) patients is considered the most effective strategy in low prevalence settings for early detection of infection and disease, and for prevention of secondary TB cases. ${ }^{1,2}$ The aims of contact investigation are primarily to find and treat secondary active TB disease to disrupt ongoing transmission. Screening for latent TB infections (LTBI) is also performed enabling preventive treatment or follow-up screening to prevent future TB disease in contacts. In addition, source tracing investigations are performed around cases of extra-pulmonary TB (ETB) to find undiagnosed pulmonary TB (PTB) cases. In the Netherlands, active TB is a notifiable disease, and source and contact investigation is included in the national TB control program. Contacts are differentiated according to the 'stone in the pond' principle, a concentric circle or ring approach based on their closeness to the index, as a proxy for exposure. The national guideline defines three rings which describe close, regular and community contacts. ${ }^{3-5}$ Based on the yield of screening in the respective rings, starting with the closest ring, and the risk of transmission as estimated by the public health nurse, the investigation can be stopped or extended to include more distant contacts.

There is an extensive amount of evidence regarding the risk factors in TB transmission. These established risk factors have been used in the national guidelines for many years. Factors such as the age of the index patient and contact, duration and intensity of the contact, bacteriological status of the index patient, and ethnic background of the index patient are all know to be important, but the relative importance of these factors has not been established for contact investigations in the Netherlands. ${ }^{6-9}$ Improved knowledge of risk factors determining the chance of contacts being infected with TB would strengthen contact investigations by improving prioritization so high-risk contacts can be targeted first.

The Municipal Public Health Service (MPHS) of Rotterdam, the Netherlands, investigates hundreds of contacts of active TB patients each year. This has resulted in a unique database that, unlike other known registries, includes information on all screened contacts, not just those who were found to have LTBI or TB. This database of index patients and their contacts offers an excellent opportunity for an in-depth evaluation of contact investigations in the control of TB. In this study, we set out to identify risk factors for TB transmission among contacts of TB patients, in order to strengthen the evidence base of the current national contact investigation policy in the Netherlands. ${ }^{10}$

\section{Materials and Methods}

The MPHS in Rotterdam is responsible for TB control in the larger Rotterdam-Rijnmond area, with a population of 1.2 million. The MPHS registers all TB control activities, including contact investigations. Since 2001, data on source tracing and contact investiga-
Correspondence: Jesse Eduard Verdier Department of Public Health, Erasmus MC University Medical Center Rotterdam, P0 Box 2040, 3000 CA Rotterdam, The Netherlands.

Tel. +31.107043732/7038460 - Fax: +31.10 7038475 . E-mail: j.verdier@erasmusmc.nl

Key words: contact investigations, epidemiological risk factors, tuberculosis transmission, tuberculosis screening.

Acknowledgments: this study was funded by ZonMw, the Netherlands organization for health research and development (grant n. 125010010). We would like to thank Gerard de Vries for his advice and input for the article, and for the tremendous effort he put into building this comprehensive database.

Funding: ZonMw, the Netherlands organization for health research and development. Grant $n$. 125010010 .

Conflict of interest: the authors report no conflicts of interest.

Received for publication: 1 September 2011.

Revision received: 19 December 2011.

Accepted for publication: 12 January 2012.

This work is licensed under a Creative Commons Attribution NonCommercial 3.0 License (CC BYNC 3.0).

(C) Copyright J.E. Verdier et al., 2012

Licensee PAGEPress, Italy

Infectious Disease Reports 2012; 4:e26

doi:10.4081/idr.2012.e26

tions have been stored systematically in a database. Our study includes data on the index cases and their contacts over the period 20012006.

Contact investigations are conducted according to indications of infectiousness of the index case, such as the anatomical location of TB disease, the bacteriological status, and estimated patient and/or doctor's delay. A public health nurse interviews the index to determine which (if any) contacts should be investigated according to the 'stone in the pond' principle of concentric rings, where the ring can be seen as a proxy for exposure to the index patient in the case of PTB. The first ring (ring 1) contains close contacts, the second ring (ring 2) contains regular contacts, while the third ring (ring 3) contains community contacts. This classification is based on the frequency and duration of exposure, and physical aspects of the area where the exposure takes place (in particular, compartment size and ventilation).,3,411,12

The MPHS databases on the index patients and contacts were combined and linked to create a new database of screened contacts to which the recorded characteristics of the index 
patient were added. For the contacts we included age, gender, date of screening, tuberculin skin test (TST) and/or chest X-ray (CXR). For the index patients we included the type of TB disease, age, ethnic background, sensitivity of the TB strain and the diagnostic steps that were taken. A positive TST was defined as an induration of $10 \mathrm{~mm}$ or more to $0.1 \mathrm{~mL}$ of purified protein derivative, measured 48 and $72 \mathrm{~h}$ after TST. Positive TST results were followed up by CXR to screen for abnormalities indicating pulmonary TB. Contacts with a history of $\mathrm{TB}$, a previous TST induration of $10 \mathrm{~mm}$ or more, evidence of BCG vaccination or birth before 1945 were only screened by CXR, as a TST would be unable to provide evidence of a recent infection in these contacts. In the Netherlands, universal BCG vaccination for the general population has never been implemented, and is only given to travellers to endemic countries or children of parents from endemic countries. When TST was positive, but clinical and radiological signs of active TB disease were absent, the final LTBI diagnosis was made by a TB physician. ${ }^{12}$

Statistical analysis was divided into two parts. The first part determined the risk factors for being diagnosed with LTBI using a TST. The second part aimed at determining the risk factors for being diagnosed with active TB. The outcome of interest in the first analysis regarding TST tested contacts was a positive TST. In the second analysis, the outcome of interest was defined as an abnormal CXR with, if possible, bacteriological confirmation of active TB. Both analyses were performed with the contacts as the unit of analysis and stratified by ring. Univariate logistic regression was performed to estimate the effect of the characteristics on the individual contact investigation outcomes. Variables with $\mathrm{P}<0.2$ in the univariate logistic model were then included in multivariate logistic regression. We used a backward-stepwise selection based on the loglikelihood ratios and excluded variables with $P>0.05$. Covariance and interactions were evaluated for the final models. Exceptionally large contact investigations were excluded from the dataset, after which the analysis was performed again to ensure these large contact subpopulations did not weigh too heavily in the regression analyses. PASW statistics software 17.0.3 (SPSS Inc., IBM, Chicago, USA) was used for the analyses.

\section{Results}

Between 2001 and 2006, a total of 1,165 index patients with active TB were registered. Of these, 731 led to investigation of at least one contact (range 1-1,810 contacts; median 6; $\mathrm{IQR}=3.22)$. Together, these 731 index patients had 21,540 contacts participating in source tracing or contact investigations (Figure 1). Their general characteristics are shown in Table 1 and in more detail in the Appendix
(Supplementary Tables 1-5). Of the contacts, 12,698 (59\%) were eligible (e.g. not previously vaccinated with BCG) and tested for latent TB infections with a TST. The database contained 20,494 unique contacts, indicating that 1,046 of the investigations (4.9\%) were performed in contacts who had participated in an earlier contact investigation between 2001 and 2006 . The percentage of TB infected contacts among those tested with TST was almost halved when we compared regular contacts with close contacts (56\%) and community contacts with regular contacts (50\%). For active TB among all contacts, the decrease is even stronger; $21 \%$ for regular contacts compared with close contacts, and $11 \%$ comparing community contacts with regular contacts.

Of the 12,698 contacts tested with TST, 1,091 were diagnosed with LTBI while the TST follow up with CXR revealed signs of active TB in 49. Table 2 shows the multivariate regression results for the three rings (details are to be found in the Appendix, Supplementary Tables $1-3)$. Older age (10-year intervals) of the contact increases the risk of having a positive TST in all rings, with odds ratios (OR) of 1.12, 1.17 and 1.32 for the first, second and third ring, respectively. The relationship between the index and the contacts indicates that residential or family relationships are at the highest risk of having a positive TST, while work or education related contact results in a lower chance of transmission, although the differences are not always statistically significant

Table 1. Characteristics of the contacts included in the source tracing and contact investigations 2001-2006 performed by the Municipal Public Health Service Rotterdam-Rijnmond, the Netherlands. Contacts are stratified by ring according to a concentric circle approach, as specified in the national guidelines for contact investigations in the Netherlands. Ring 1 contains close contacts, ring 2 contains regular contacts, while ring 3 contains community contacts.

\begin{tabular}{|c|c|c|c|c|c|}
\hline & $\begin{array}{c}\text { Ring } 1, \text { TST } \\
\text { tested }\end{array}$ & $\begin{array}{c}\text { Ring } 2, \text { TST } \\
\text { tested }\end{array}$ & $\begin{array}{c}\text { Ring } 3, \text { TST } \\
\text { tested }\end{array}$ & Ring 1 all & Ring 2 all \\
\hline Total n. contacts & 1697 & 6232 & 4769 & 3296 & 10967 \\
\hline Postives* & $16.0 \%$ & $9.0 \%$ & $4.3 \%$ & $9.6 \%(1.5 \% *)$ & $5.7 \%(0.3 \% *)$ \\
\hline Male gender & $50.1 \%$ & $51.1 \%$ & $50.1 \%$ & $51.9 \%$ & $50.7 \%$ \\
\hline Age of contact, median (interquartile range) & $25(14-39)$ & $32(21-43)$ & $28(20-40)$ & $28(15-42)$ & $34(23-46)$ \\
\hline $\begin{array}{l}\text { Relationship between contact and index } \\
\text { Residential/family } \\
\text { Work/education } \\
\text { Leisure time/other }\end{array}$ & $\begin{array}{c}27.7 \% \\
64.4 \% \\
4.4 \%\end{array}$ & $\begin{array}{l}18.5 \% \\
56.8 \% \\
21.8 \%\end{array}$ & $\begin{array}{l}1.0 \% \\
48.8 \% \\
29.2 \%\end{array}$ & $\begin{array}{l}29.7 \% \\
61.6 \% \\
5.0 \%\end{array}$ & $\begin{array}{l}20.7 \% \\
54.6 \% \\
21.9 \%\end{array}$ \\
\hline Unknown/censored & $3.5 \%$ & $2.9 \%$ & $21.0 \%$ & $3.7 \%$ & $2.7 \%$ \\
\hline Age of index, median (interquartile range) & $31(23-47)$ & $34(25-44)$ & $33(19-44)$ & $33(23-47)$ & $34(25-47)$ \\
\hline $\begin{array}{l}\text { Contacts investigated by location and bacteriological status of index } \\
\text { PTB AFB+ } \\
\text { PTB AFB- } \\
\text { ETB }\end{array}$ & $\begin{array}{l}75.0 \% \\
15.5 \% \\
9.5 \%\end{array}$ & $\begin{array}{l}87.5 \% \\
9.7 \% \\
2.8 \%\end{array}$ & $\begin{array}{l}94.0 \% \\
5.9 \% \\
0.1 \%\end{array}$ & $\begin{array}{l}67.6 \% \\
18.8 \% \\
13.6 \%\end{array}$ & $\begin{array}{l}87.2 \% \\
9.4 \% \\
3.4 \%\end{array}$ \\
\hline $\begin{array}{l}\text { Contacts investigated by index cases and specific ethnic background } \\
\text { Dutch } \\
\text { Western } \\
\text { Non-western }\end{array}$ & $\begin{array}{c}63.6 \% \\
0.8 \% \\
35.7 \%\end{array}$ & $\begin{array}{c}51.8 \% \\
1.1 \% \\
47.1 \%\end{array}$ & $\begin{array}{c}73.5 \% \\
0.1 \% \\
26.4 \%\end{array}$ & $\begin{array}{c}52.0 \% \\
0.8 \% \\
47.2 \%\end{array}$ & $\begin{array}{c}47.2 \% \\
1.2 \% \\
51.7 \%\end{array}$ \\
\hline
\end{tabular}

TST, tuberculin skin test; PTB, pulmonary tubercolosis; ETB, extra-pulmonary tubercolosis. *Percentage of people found positive in the diagnostic test, confirmed by the tubercolosis (TB) physician. Percentage includes the number of latent TB infections (LTBI) cases in tuberculin skin test-tested persons, the percentage of TB and LTBI for the column with all contacts. For columns including all ring 1 and 2 contacts, the percentage in brackets represents active TB percentage. 
among the types of relationships or for every ring. The diagnosis of the index is a strong predictor for contacts with positive TST: smear positive PTB (PTB $\mathrm{AFB}+$ ) results significantly more often in the diagnosis of LTBI in contacts compared to smear negative PTB (PTB AFB-) or ETB in ring 1, but the differences between $\mathrm{PTB}+$ and PTB- decrease in ring 2 and there is no significant difference between them in ring 3. Additionally, the ethnic background of the index patient is statistically significant in rings 1 and 3 , but not in ring 2 . As far as investigation characteristics are concerned, the infection fraction of rings closer to the index is a strong predictor for the more remote rings. For ring 2, OR is 3.19 (95\% $\mathrm{CI}: 2.4-4.2)$ in the multivariate analysis, with the fraction positives within an investigation as risk factor. The correlation is more pronounced in ring 3 , in which the positive fraction of ring 2 gives an OR of 9.22 (95\% CI:3.37-25.2).

Among the 21,540 contacts, 91 cases of active TB were diagnosed. Of these 91 cases, 2 were found by source tracing, meaning that these 2 screen-detected PTB cases were likely

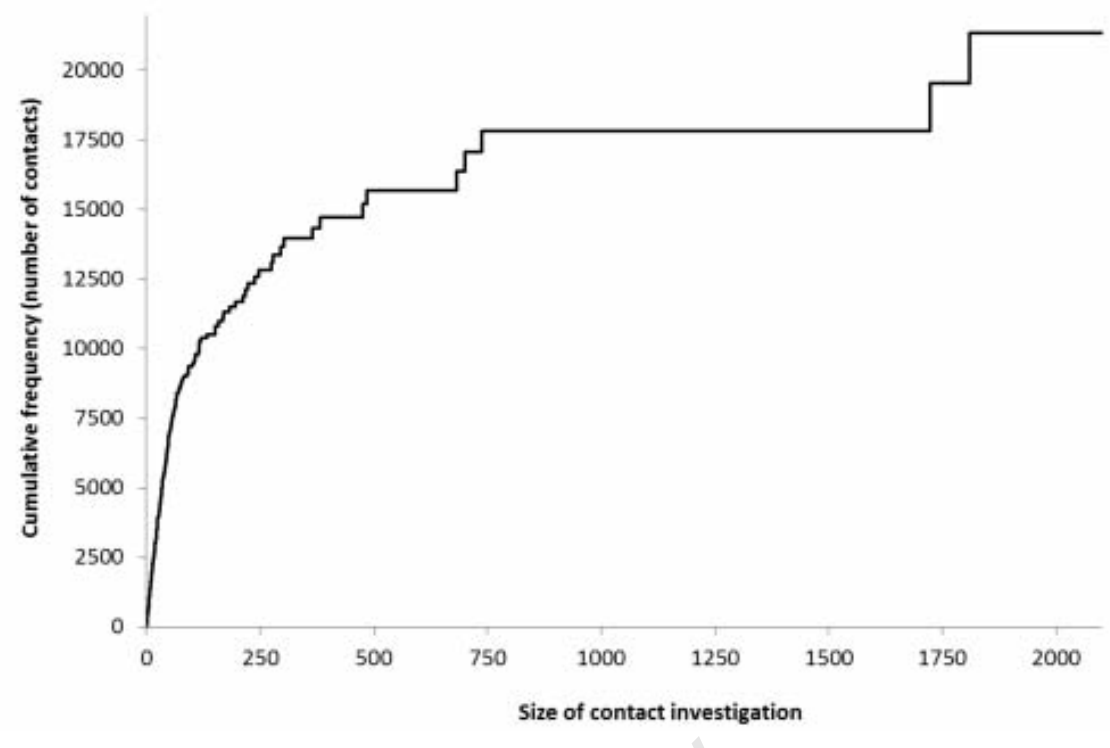

Figure 1. Cumulative number of contacts screened over the period 2001-2006 in source tracing or contact investigations in the Rotterdam-Rijnmond area plotted against the size of the individual contact investigations.

Table 2. Odds ratios and their corresponding $95 \%$ confidence intervals for all significant factors in source tracing and contact investigations among contacts of TB patients from the five multivariate logistic regression models. 'Gender' did not reach significance in any of the models and is not, therefore, shown. Ring 1 contains close contacts, ring 2 contains regular contacts, ring 3 contains community contacts.

\begin{tabular}{|c|c|c|c|c|c|}
\hline & \multicolumn{3}{|c|}{ TST tested contacts } & \multicolumn{2}{|c|}{ TST and/or CXR screened contacts } \\
\hline & Ring 1 LTBI & Ring 2 LTBI & Ring 3 LTBI & Ring 1 TB & Ring 2 TB \\
\hline Age of contact (10-year interval continuous) & $1.12(1.03-1.22)$ & $1.17(1.09-1.24)$ & $1.32(1.19-1.47)$ & $0.71(0.59-0.85)$ & \\
\hline $\begin{array}{l}\text { Relationship } \\
\text { Residential/family } \\
\text { Work/education } \\
\text { Leisure time/other } \\
\text { Unknown/censored }\end{array}$ & $\begin{array}{c}1.00 \\
0.55(0.41-0.74) \\
0.57(0.28-1.17) \\
0.57(0.27-1.19)\end{array}$ & $\begin{array}{c}1.00 \\
0.51(0.41-0.65) \\
0.77(0.60-0.99) \\
1.59(1.05-2.41)\end{array}$ & & & $\begin{array}{c}1.00 \\
0.25(0.12-0.55) \\
0.63(0.29-1.41) \\
0.61(0.08-4.68)\end{array}$ \\
\hline Age of index (10-year interval) & $0.83(0.76-0.92)$ & & $1.28(1.14-1.44)$ & & $0.78(0.61-1.00)$ \\
\hline $\begin{array}{l}\text { Ethnic background } \\
\text { Dutch } \\
\text { Western } \\
\text { Non-western }\end{array}$ & $\begin{array}{c}1.00 \\
2.08(1.40-3.09) \\
1.70(1.28-2.27)\end{array}$ & & $\begin{array}{c}1.00 \\
2.41(1.29-4.51) \\
3.44(2.41-4.90)\end{array}$ & & \\
\hline $\begin{array}{l}\text { Bacteriological status index } \\
\text { PTB AFB+ } \\
\text { PTB AFB- } \\
\text { ETB }\end{array}$ & $\begin{array}{c}1.00 \\
0.31(0.19-0.50) \\
0.49(0.29-0.85)\end{array}$ & $\begin{array}{c}1.00 \\
0.98(0.72-1.32) \\
0.25(0.09-0.68)\end{array}$ & & $\begin{array}{c}1.00 \\
0.21(0.09-0.58) \\
0.09(0.02-0.39)\end{array}$ & \\
\hline $\begin{array}{l}\text { Drug resistance } \\
\text { Susceptible } \\
\text { Resistant }\end{array}$ & & & $\begin{array}{c}1.00 \\
4.61(1.90-11.2)\end{array}$ & & \\
\hline $\begin{array}{l}\text { BAL performed } \\
\text { Yes } \\
\text { No }\end{array}$ & & $\begin{array}{c}0.080(0.64-0.99) \\
1.00\end{array}$ & & & \\
\hline Investigation size (100-person interval) & & $1.05(1.01-1.08)$ & & $0.19(0.06-0.58)$ & \\
\hline $\begin{array}{l}\text { Quarter of starting the investigation } \\
\text { First } \\
\text { Second } \\
\text { Third } \\
\text { Fourth }\end{array}$ & & & & $\begin{array}{c}1.00 \\
2.26(0.84-6.07) \\
4.20(1.60-11.0) \\
3.92(1.54-9.97)\end{array}$ & \\
\hline Fraction infected in ring 1 & $\mathrm{NA}^{*}$ & $3.19(2.40-4.23)$ & $\mathrm{NA}^{*}$ & $\mathrm{NA}^{*}$ & $3.67(1.44-9.38)$ \\
\hline Fraction infected in ring 2 & $\mathrm{NA}^{*}$ & $N A^{*}$ & $9.22(3.37-25.2)$ & $\mathrm{NA}^{*}$ & $\mathrm{NA}^{*}$ \\
\hline
\end{tabular}

TST, tuberculin skin test; CXR, chest X-ray; LTBI, latent tubercolosis infections; TB, tubercolosis; PTB, pulmonary tubercolosis; ETB, extra-pulmonary tubercolosis; BAL, benzaldehyde. * NA, not applicable, as only more proximal fraction of infected contacts within a source and contact investigation was entered into the multivariate regression analysis. 
the first patients in the cluster. The correlation observed for the type of relationship between index and contact in the LTBI screening analysis was also seen for contacts diagnosed with active TB; work or education related contact has a lower relative risk of being diagnosed with TB, compared to residential, family, leisure time, or other contacts. For ring 1 , the bacteriological status of the index is a strong predictor, with an OR of 0.21 for PTB- and 0.09 for ETB, compared to PTB+. The infection fraction in ring 1 is also a predictor for being diagnosed with TB in ring2, with an OR of 3.67 (95\% CI:1.4-9.4). The age of the contacts showed an inverse relationship to active TB, compared to the association found for LTBI, as $40 \%$ of the TB cases were in children aged 14 years or under with an odds ratio of 0.70 in the multivariate model in ring 1 , with 16 cases of active TB disease in young children aged 5 years or under. It was not possible to analyze the community contacts because only 2 cases of active TB were found among the 7,277 contacts in this ring. After exclusion of the largest 5 contact investigations ( $26 \%$ of all contacts in the study) the direction of the associations did not change (data not shown). Details of the analysis of the close and regular contacts are shown in the Appendix (Supplementary Tables 4 and 5).

\section{Discussion}

Between 2001 and 2006, at least one contact was screened of 731 index patients, resulting in a total of 21,540 contacts. These contact investigations revealed 91 cases of active TB. The screening of 12,698 contacts who were eligible for TST testing led to the detection of 1,091 LTBI cases. Important risk factors were age of the contact, age of the index patient and the relationship to the index. Infections among regular (ring 2) or incidental and community (ring 3 ) contacts were strongly associated with a higher fraction of infected close (ring 1) or regular (ring 2) contacts, respectively.

Our study is based on data from routine investigations, which has implications for the interpretation of the findings. The records of all index patients and their contacts (if any) were available, enabling comparison between those with positive and those with negative contact investigation outcomes. Although all contacts who participated and all investigations were documented, it is difficult to determine how many contacts were missed. A fraction of contacts will be missed by the MPHS, despite the extensive effort to screen all reported or possibly exposed contacts. Some groups are not included in routine contact investigations, such as the homeless or illicit substance users, because there is a separate regular screening program for these subpopulations. ${ }^{13}$ A recent study using data from the Dutch TB surveillance register showed that contacts of immigrant cases were less often investigated. ${ }^{14}$ Despite possible incompleteness of the study population, this database provides an excellent opportunity to determine risk factors in contact investigations because the Netherlands is one of few countries where contact investigations are fully documented, out of the thirteen countries known to investigate contacts of all possibly infectious index patients. ${ }^{15,16}$

Our study confirmed that three main factors are significantly associated with TB transmission. ${ }^{5}$ Firstly, close contacts (ring 1 ) had a higher risk of infection and TB disease than regular (ring 2) and community (ring 3 ) contacts. Although more refined proxies for exposure are possible, the currently used ring approach has proven to be adequate in discriminating between contact levels. It is unknown how much could be gained by using data underlying this current proxy, such as actual duration and frequency of contact, and the environmental characteristics of the place of contact. Unfortunately, a more precise registration of such indicators of contact level is not practical under routine control conditions. Secondly, the contagious status of the index patient, in this study recorded as bacteriological status of the index patient in combination with the infection fraction in closer contact rings, was strongly associated with the number of infections among distant contacts. It is unclear whether this effect is fully attributable to recent transmission or is because the screened contacts are part of a subpopulation with a higher risk of infection. This is one of the limitations of using the TST in contact investigations. Finally, increasing age of the contacts was associated with the probability of a TB infection. This is understandable from the perspective that with age, the time that an individual has been at risk of coming into contact with TB (and other cross-reacting non-TB mycobacteria) accumulates. Young children (age $<5$ years) are more at risk for progression to active TB, which is also recognized in this study, but in our analysis this was only proven in ring 1 . These three factors have already been well established as risk factors for TB in contacts and are included in the national guidelines for source tracing and contact investigations in the Netherlands. Similar results have been reported in evaluations of contact investigations in comparable settings and one systematic review, while one study did not find an association between the closeness to the index and symptomatic PTB after confirming transmission through DNA fingerprinting. ${ }^{17-20}$

The study was carried out before the application of the interferon- $\gamma$ assay blood test
(IGRA) in the Netherlands. Later studies in comparable settings show that the IGRA test has a higher specificity than TST and thereby reduces the number of persons who would have received prophylactic treatment in case of a false-positive TST. ${ }^{21-23}$ The IGRA also helps to avoid LTBI treatment for non-tuberculosis mycobacteria infections. ${ }^{24,25}$ We do not expect that the results would change drastically if the IGRA test were applied instead of the TST. There is no indication that the IGRA test responds differently in the risk groups that we established in this study, other than detecting additional old infections in BCG vaccinated persons from endemic countries. In this respect, we would like to point out that the goal of contact investigations is primarily to prevent large clusters by stopping ongoing transmission.

This study focused on a population of contacts participating in source tracing and contact investigations. We identified important risk factors for the diagnosis of TB among contacts of TB patients, based on routine practice in a low prevalence setting, and established that the findings basically match the current national guidelines. The age of the patients and their contacts, the number of infections among close contacts, the type of contact relationship, and the diagnosis of the index patient are all associated with LTBI and TB in contacts. These risk factors emphasize the importance of including personal and interpersonal factors in decision making regarding contact investigations.

\section{References}

1. Dasgupta K, Menzies D. Cost-effectiveness of tuberculosis control strategies among immigrants and refugees. Eur Respir J 2005;25:1107-16.

2. Underwood BR, White VL, Baker T, et al. Contact tracing and population screening for tuberculosis--who should be assessed? J Public Health Med 2003;25:59-61.:

3 . Veen J. Tuberculosis in a low prevalence country: A wolf in sheep's clothing. Bull Int Union Tuber Lung Dis 1991;66:203-5.

4. Veen J. Microepidemics of tuberculosis: the stone-in-the-pond principle. Tuber Lung Dis 1992;73:73-6.

5. Van Geuns AH. Contact onderzoek. T d Tuberculose 1972;70-5.

6. MacIntyre CR, Plant AJ. Impact of policy and practice on the effectiveness of contact screening for tuberculosis. Prev Med 1998;27:830-7.

7. Borgdorff MW, Nagelkerke NJ, de Haas PE, van Soolingen D. Transmission of Mycobacterium tuberculosis depending on the age and sex of source cases. Am J 
Epidemiol 2001;154:934-43.

8. Menzies D. Issues in the management of contacts of patients with active pulmonary tuberculosis. Can J Public Health 1997;88:197-201

9. Borgdorff MW, Nagelkerke N, van Soolingen D, et al. Analysis of tuberculosis transmission between nationalities in the Netherlands in the period 1993-1995 using DNA fingerprinting. Am J Epidemiol 1998;147:187-95.

10. Ansari S, Thomas S, Campbell IA. Refined tuberculosis contact tracing in a low incidence area. Respir Med 1998;92:1127-31.

11. Pool IA. Het contactonderzoek, pijler in de tuberculosebestrijding (National guideline). The Hague: KNCV Tuberculosis Foundation; 2004.

12. Erkens CG, Kamphorst M, Abubakar I, et al. Tuberculosis contact investigation in low prevalence countries: a European consensus. Eur Respir J 2010;36:925-49.

13. de Vries G, van Hest RA. From contact investigation to tuberculosis screening of drug addicts and homeless persons in Rotterdam. Eur J Public Health 2006;16: 133-6.

14. Mulder C, Erkens CGM, Kouw PM, et al. Missed opportunities in tuberculosis con- trol in The Netherlands due to prioritization of contact investigations. Eur J Public Health 2011;10:1093.

15. Bothamley GH, Ditiu L, Migliori GB, Lange $\mathrm{C}$; TBNET contributors. Active case finding of tuberculosis in Europe: a Tuberculosis Network European Trials Group (TBNET) survey. Eur Respir J 2008;32:1023-30.

16. Mulder C, Klinkenberg E, Manissero D. Effectiveness of tuberculosis contact tracing among migrants and the foreign-born population. Euro Surveill 2009;14:19153.

17. Behr MA, Hopewell PC, Paz EA, et al. Predictive value of contact investigation for identifying recent transmission of Mycobacterium tuberculosis. Am J Respir Crit Care Med 1998;158:465-9.

18. Marks SM, Taylor Z, Qualls NL, et al. Outcomes of contact investigations of infectious tuberculosis patients. Am J Respir Crit Care Med 2000;162:2033-8.

19. Langenskiold E, Herrmann FR, Luong BL, et al. Contact tracing for tuberculosis and treatment for latent infection in a low incidence country. Swiss Med Wkly 2008;138:78-84.

20. Nava-Aguilera E, Andersson N, Harris E, et al. Risk factors associated with recent transmission of tuberculosis: systematic review and meta-analysis. Int $\mathrm{J}$ Tuberc Lung Dis 2009;13:17-26.

21. Janssens JP, Roux-Lombard P, Perneger T, et al.Contribution of a IFN-gamma assay in contact tracing for tuberculosis in a lowincidence, high immigration area. Swiss Med Wkly 2008;138:585-93.

22. Diel R, Loddenkemper R, Meywald-Walter $\mathrm{K}$, et al. Comparative performance of tuberculin skin test, QuantiFERON-TBGold In Tube assay, and T-Spot.TB test in contact investigations for tuberculosis. Chest 2009;135:1010-8.

23. Kowada A, Hamada Y, Aoki M, et al. Utility of QuantiFERON TB-2G for tuberculosis contact investigations in public health services. Jap J Pub Health 2007;54:434-9.

24. Diel R, Nienhaus A, Lange C, MeywaldWalter K. Tuberculosis contact investigation with a new, specific blood test in a low-incidence population containing a high proportion of BCG-vaccinated persons. Respir Res 2006;17:77.

25. Mazurek GH, Jereb J, Lobue $P$, et al. Guidelines for using the QuantiFERON-TB Gold test for detecting Mycobacterium tuberculosis infection, United States MMWR. Recomm Rep 2005;16:49-55 